\title{
Improved Scalability of Neuron-Based Phenotypic Screening Assays for Therapeutic Discovery in Neuropsychiatric Disorders
}

\author{
Timothy P. Spicer ${ }^{a} \quad$ Christopher Hubbs $^{b} \quad$ Thomas Vaissiere $^{b} \quad$ Deanna Collia $^{a}$ \\ Camilo Rojas $^{\mathrm{b}}$ Murat Kilinc $^{\mathrm{b}}$ Kyle Vick $^{\mathrm{b}, \mathrm{d}}$ Franck Madoux $^{\mathrm{a}, \mathrm{c}}$ \\ Pierre Baillargeon $^{a}$ Justin Shumate ${ }^{a}$ Kirill A. Martemyanov ${ }^{b}$ Damon T. Page ${ }^{b}$ \\ Sathya Puthanveettil ${ }^{b}$ Peter Hodder ${ }^{a, c}$ Ronald Davis ${ }^{b}$ Courtney A. Miller ${ }^{a, b}$ \\ Louis Scampavia ${ }^{a}$ Gavin Rumbaugh ${ }^{a, b}$ \\ Departments of a Molecular Medicine and ${ }^{\mathrm{b}}$ Neuroscience, The Scripps Research Institute, Jupiter, FL, \\ ${ }^{\mathrm{C}}$ Amgen, Thousand Oaks, CA, and ${ }^{\mathrm{d}}$ Aerie Pharmaceuticals, Durham, NC, USA
}

\section{Keywords}

Autism $\cdot$ Molecular imaging $\cdot$ Neuroscience .

Synaptogenesis - Therapeutic discovery

\begin{abstract}
There is a pressing need to improve approaches for drug discovery related to neuropsychiatric disorders (NSDs). Therapeutic discovery in neuropsychiatric disorders would benefit from screening assays that can measure changes in complex phenotypes linked to disease mechanisms. However, traditional assays that track complex neuronal phenotypes, such as neuronal connectivity, exhibit poor scalability and are not compatible with high-throughput screening (HTS) procedures. Therefore, we created a neuronal phenotypic assay platform that focused on improving the scalability and affordability of neuron-based assays capable of tracking disease-relevant phenotypes. First, using inexpensive laboratory-level automation, we industrialized primary neuronal culture production, which enabled the creation of scalable assays within functioning neural networks. We then developed a panel of phenotypic assays based on culturing of pri-
\end{abstract}

mary neurons from genetically modified mice expressing HTS-compatible reporters that capture disease-relevant phenotypes. We demonstrated that a library of 1,280 compounds was quickly screened against both assays using only a few litters of mice in a typical academic laboratory setting. Finally, we implemented one assay in a fully automated high-throughput academic screening facility, illustrating the scalability of assays designed using this platform. These methodological improvements simplify the creation of highly scalable neuron-based phenotypic assays designed to improve drug discovery in CNS disorders. @ 2017 S. Karger AG, Basel

\section{Introduction}

The drug discovery pipelines for brain disorders are relatively dry compared to those for diseases linked to other organs $[1,2]$. An approach to rejuvenate these pipe-

T.P. Spicer, C. Hubbs, and T. Vaissiere contributed equally to this paper.

\section{KARGER}

(C) 2017 S. Karger AG, Basel

E-Mail karger@karger.com

www.karger.com/mnp
Gavin Rumbaugh

Department of Neuroscience, The Scripps Research Institute

130 Scripps Way, \#3B3

Jupiter, FL 33458 (USA)

E-Mail grumbaug@scripps.edu 
lines is to create high-throughput screening (HTS)-capable assays that model CNS disease-relevant phenotypes as closely as possible [3-7]. Immortalized cell lines serve as the standard for phenotypic screening because of their scalability and adaptability to different types of assays. However, many CNS disease phenotypes can only be recapitulated within mature neural networks and, therefore, cannot be modeled effectively in nonneuronal cells or even induced pluripotent stem cell (iPSC)-derived neurons. Indeed, while iPSCs and subsequent derivation to neurons or organoids is a promising technology, significant questions remain with respect to the scalability, reliability, and reproducibility of this technology with respect to CNS drug discovery.

Rodent primary neurons remain the gold standard source material for in vitro neurobiological investigations. Primary neurons cultured from newborn animals differentiate into mature neurons that connect to each other and form functioning neural networks. As a result, they are well suited for the development of assays that seek to capture more complex phenotypes believed to contribute to disorders of the brain. Historically, there have been significant challenges associated with harvesting and consistently growing primary neurons on a scale compatible with HTS. Recently, screening assays have been created that take advantage of complex phenotypes expressed by primary neurons $[8,9]$. However, these assays have a relatively low throughput and are not particularly scalable to large screening projects that routinely use libraries of 20,000-100,000 unique probes. Limited scalability in current primary neuron-based phenotypic assay strategies is caused by the use of low-density (96-well) assay plates combined with laborious procedures to label assay endpoints. The poor scalability of neuron-based phenotypic assays is a significant roadblock in NSD-related therapeutic discovery. Screening projects provide initial leads for therapeutic development. Thus, increasing assay scalability enables the selection of larger and more diverse libraries for screening, which increases the chances of success in these projects. To improve the scalability of neuron-based phenotypic screening assays, we combined genomic targeting of fluorescent assay reporters in rodents with implementation of low-cost automation and high-density neuronal cultures. These innovations enabled the creation of multiple neuron-based phenotypic assays that demonstrated significantly improved scalability over current platforms. These optimized workflows are expected to improve the success rate of lead identification for therapeutic discovery related to neuropsychiatric disorders.

\section{Methods}

Mouse Lines

For most experiments, primary neurons were derived from offspring resulting in a cross between male homozygous AI34D (JAX\#012570) and female WT C57/BL6j mice. In one study, male homozygous SOM-ires-Cre mice (JAX\#013044) were crossed with homozygous female AI34D mice to induce SynaptophysintdTomato in a subset of GABAergic neurons. The Syngap1 reporter line (Rum3) was generated by the insertion of the IREStdTomato construct into the exon 21 of Syngap1. This insertion resulted in the expression of Syngap and tdTomato from a bicistronic mRNA. The investigation of the temporal expression of Syngap and tdTomato was done using a pan SynGAP and a tdTomato antibody.

\section{Cell Culture Protocol for Screening Assays (96- and 384-Well}

Plates)

Forebrain dissection of $\mathrm{P} 0$ mouse pups to isolate primary cortical neurons ( 1 pup per culture plate) was performed, followed by dissociation with 20 active units/mL papain in $1 \mathrm{~mL} / \mathrm{brain}$ of dissection media for $20 \mathrm{~min}$. Cells were diluted in plating media (Neurobasal medium supplemented with $5 \% \mathrm{FBS}$ ), then robotically dispensed into 384 -well $(12,000$ cells in $80 \mu \mathrm{L} /$ well $)$ plates using an epMotion 5075 liquid handling robot. After $4 \mathrm{~h}, 80 \%$ of the plating media was replaced with feeding media (Neurobasal-A medium supplemented with $2 \%$ B-27). At 4 days in vitro (DIV4), $50 \%$ of the conditioned media was replaced with fresh feeding media containing $10 \mu \mathrm{M} 5$-fluoro- $2^{\prime}$-deoxyuridine (FUDR) to suppress the proliferation of glia. For some studies, there was a final feeding at DIV7/8. Cultures were transduced with $3 \times 10^{8}$ AAV-Cre particles/mL at DIV0 in the screening studies shown in Figure 1 and $1 \times 10^{8} \mathrm{AAV}$-Cre particles $/ \mathrm{mL}$ in the screening studies shown in Figure 2 .

\section{Cell Culture Protocol for 1,536-Well Plates}

As 1,536-well plates are not compatible with the epMotion 5075 liquid handling robot and aspiration steps are challenging, we developed an ultra-simplified procedure for culturing primary neurons in this format. The dissection and dissociation protocol was the same as that for 384-well plates. However, instead of using plating media, cells were diluted in feeding media containing $10 \mu \mathrm{M}$ FUDR and then dispensed into 1,536-well (3,000 cells in $15 \mu \mathrm{L}$ per well) plates using a Mantis liquid dispensing robot (Formulatrix Inc., Bedford, MA, USA). AAV-Cre was mixed with the cell suspension immediately before the cell dispense step. No other culturing steps were necessary up to DIV12.

\section{NucBlue Live Cell Staining}

NucBlue Live Ready Probes Reagent (ThermoFisher Scientific, Waltham, MA, USA) is a room temperature-stable live cell stain that contains Hoechst 33342, a cell-permeant nuclear counterstain that emits blue fluorescence when bound to DNA. In order to minimize cell toxicity, the $1 \mathrm{X}$ protocol $(2 \mathrm{drops} / \mathrm{mL})$ was diluted to $0.1 \mathrm{X}$ ( 2 drops $/ 10 \mathrm{~mL}$ ). At DIV7, the cells were fed with $50 \%$ feeding media $+0.1 \mathrm{X}$ NucBlue prior to imaging.

\section{Phase Contrast Cell Count Imaging}

For DIV12-DIV14 experiments, we switched to phase contrast cell count imaging at DIV7 in order to preserve cell viability over 


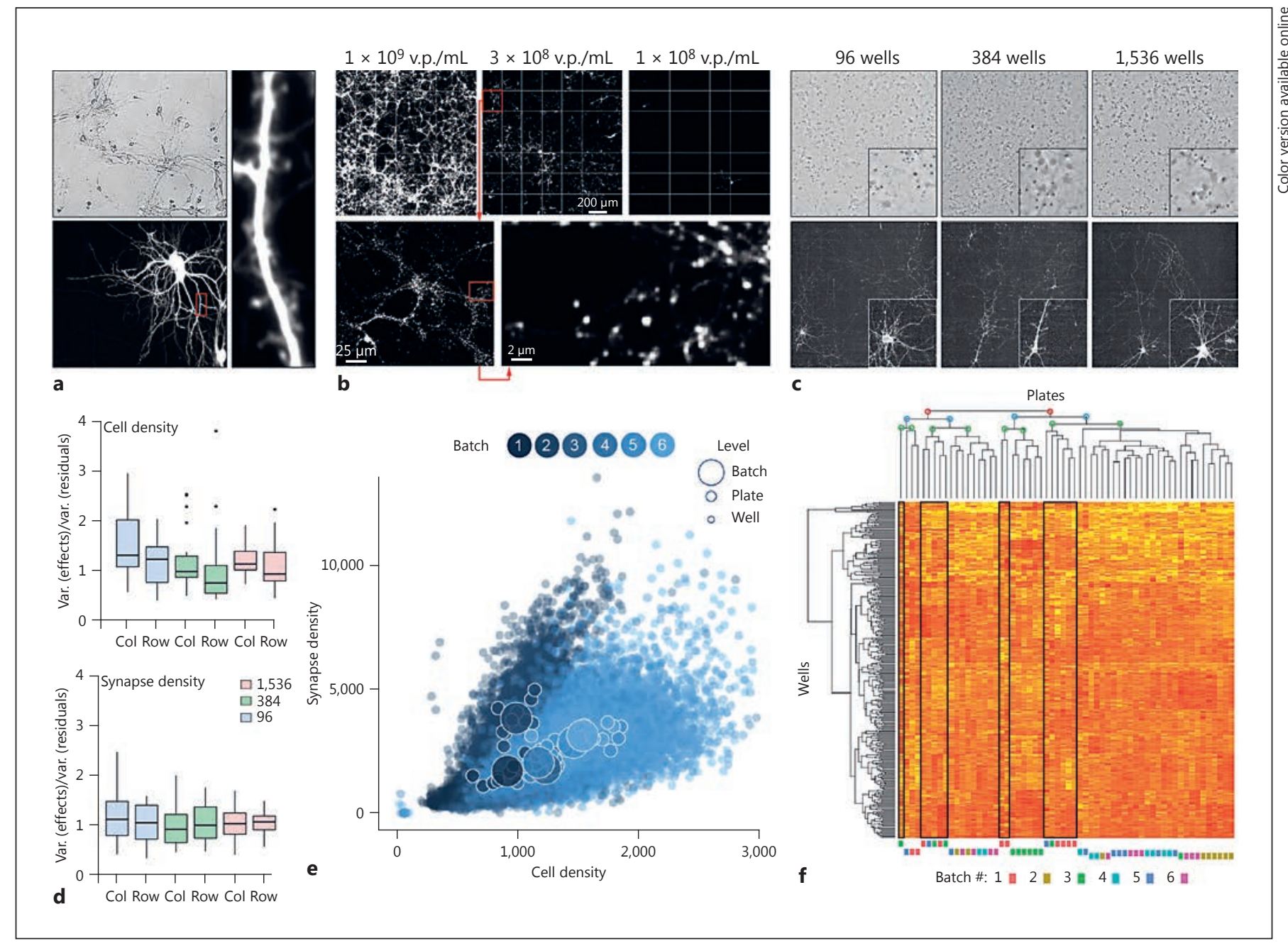

Fig. 1. Primary neuron cultures grown for screening purposes are scalable and reliable. a Primary mouse neurons cultured in a 96well assay plate transfected with a cDNA for tdTomato and imaged at DIV12. b Synaptophysin-TdTomato expression from AI34D mice 7-9 days after plating. AAV-Cre was added at various titers at plating to induce reporter expression. c Primary neuron culture and expression of reporter (as in $\mathbf{b}$ ) is scalable across three formats: 96-, 384-, and 1,536-well plates. d Homogeneity of cell number (phase contrast) and synapse density (Synaptophysin-TdTomato expression, as in c) across plate formats and within plates after me-

the experimental duration. We used the IN Cell 6000's built-in IATIA system to generate synthetic phase contrast images which were then analyzed using a custom Macro in ImageJ.

\section{Plate Imaging}

For most studies, plates were imaged with the IN Cell 6000 confocal plate reader (GE Healthcare Biosciences, Pittsburgh, PA, USA). Synaptophysin-tdTomato and NucBlue were imaged using the Cy3 and DAPI channel, respectively. For the replication compound library screen, a CellInsight ${ }^{\mathrm{TM}}$ High Content Screening dian polish procedure to estimate row and column effects at DIV7. Medians are presented within boxes for the 75th and 25th quantiles for which \pm 1.5 times the interquartile values are displayed as vertical bars; dots represent outliers. e Relationship between synapse (Synaptophysin-TdTomato expression as in c) and cell number (NucBlue staining) for 23,040 data points at DIV7 across 60 plates (384-well format) from 6 different batches. f Unsupervised clustering analysis for 384 cell density measures across 60 plates (as in e). v.p., viral particles.
Platform (ThermoFisher Scientific) was used to read Synaptophysin-tdTomato signals. For all studies that used the IN Cell 6000 reader, $1-4$ fields of view were acquired with a $10 \times$ or $20 \times$ primary magnification. Studies that used the CellInsight reader were always carried out using $20 \times$ magnification and 4 fields of view.

\section{Data Analysis for Nonscreening Data}

Column and row effects were evaluated in 96-, 384-, and 1,536well plates in which measure of synapse and cell number were acquired at DIV7. The Tukey two-way median polish procedure was 
Fig. 2. Low- and high-throughput screening against a live primary neuron-based synaptogenesis assay. a Each well of a single 384-well plate was imaged for Syn-TD puncta density at three distinct time points, which yielded two developmental epochs. Lines connect each well over time. b SynTD ratio from DIV7 to DIV9 $(n=23,040$, 60 plates with 384 wells, median $=2.5)$ or DIV 12 to DIV 14 ( $n=2,048,64$ DMSO controls from 32 plates, median =1.5). c Comparison of coefficient of variation in synapse reporter density at single time points and the ratio of detected synapses from two epochs in culture development in a 384well plate (DIV7-DIV9, estimation of variance across 384 wells per plate for 60 plates [ $n=60]$, and DIV12-DIV14, estimation of variance across 64 wells per plate for 32 plates $[n=32]$, epochs were sampled). Medians are presented within boxes for the 75th and 25th quantiles for which \pm 1.5 times the interquartile values are displayed as vertical bars; dots represents outliers. d Ratio obtained for hit identification after plate-by-plate normalization to DMSO control in a screen (s1) performed in a standard laboratory environment. Error bars represent SEM and mean of 4 replicates ( $n=256$, DMSO control and 1,280 compounds, individual DMSO replicate are represented in red). e Replicate screen (s2) performed in a $\mu \mathrm{HTS}$ environment. Compounds above 3 standard deviation of the mean of DMSO control are outlined (blue) as well as replicated hit (red) for $\mathbf{d}$ and $\mathbf{e}$. f Comparison of synapse ratio in the screens $s 1$ and s2 for three distinct AMPA receptor antagonists present in the library ( $n=4$ per compound). The gray line indicates the hit threshold. $g$ tdTomato counter screen data $(n=64,0.125 \%$ DMSO; $n=16$ per compound). DIV, days in vitro normalized to DMSO mean. Groups had equal variance (Levene's test homogeneity of variance: $\mathrm{F}[3,108]=0.296, p=0.826)$ and were not normally distributed (ShapiroWilk test $p<0.01$ ), thus statistical analysis was performed by independent two-group Mann-Whitney U test with continuity correction: DMSO versus GYKI $(\mathrm{U}=411, p=$ $0.227)$; DMSO versus CNQX $(\mathrm{U}=511, p=$ 0.977); DMSO versus NBQX ( $\mathrm{U}=334, p=$ $0.03)$. $\mathbf{d}-\mathbf{g}$ Mean and SEM are presented.

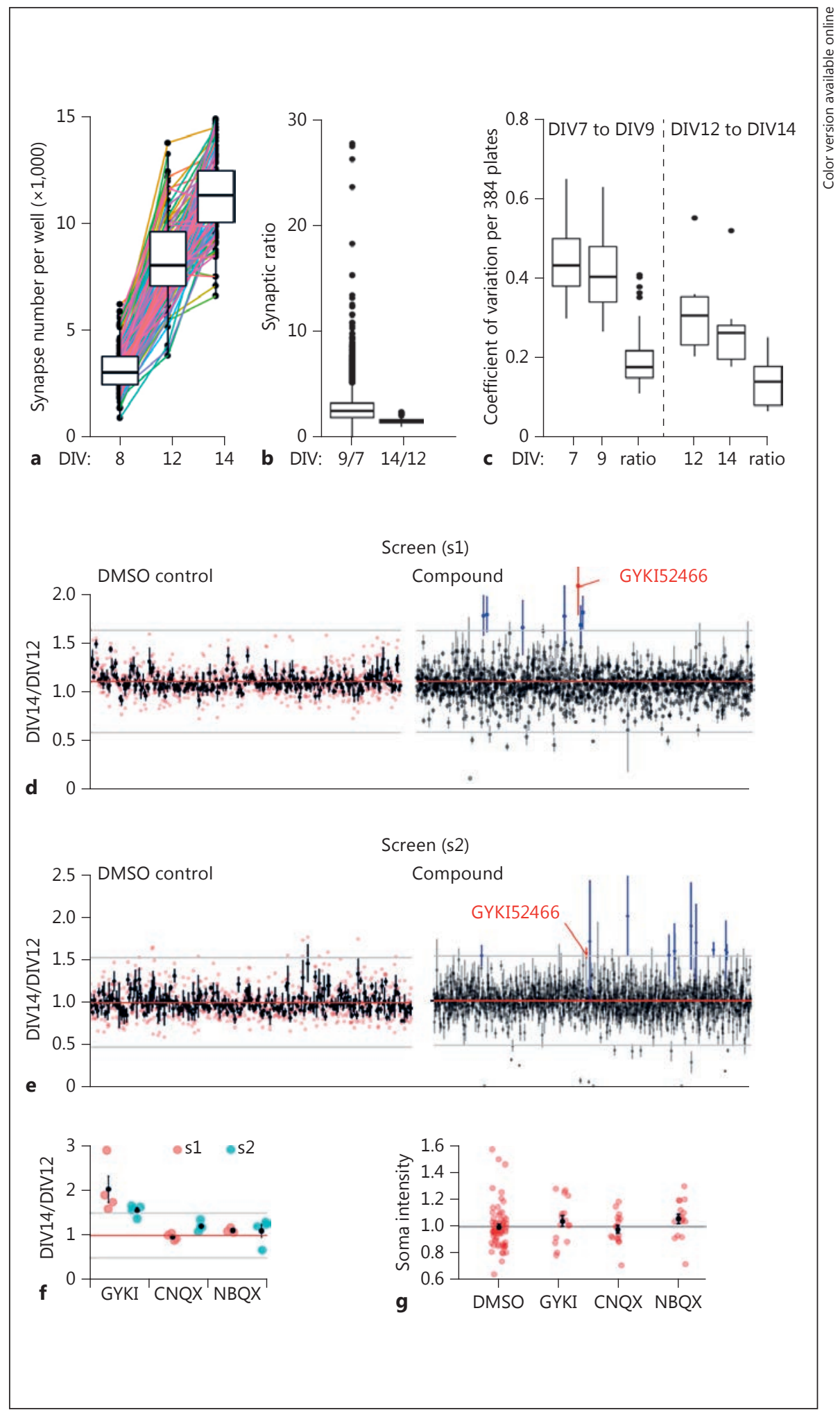


done on the three formats to estimates those position effects. The variance of the rows and the column effects were divided by the variance of all the residuals per well obtained in $\mathrm{R}$ using the medpolish function of the graphics package. For example, in 96-well plates the variance of 12 columns and 8 rows effects were divided by the variance of the 96 residuals. To understand how plates were similar after a stress test of plating six batches of ten 384-well plates per batch, an unsupervised clustering analysis was performed looking at cell densities. The similarity among the 60 plates was displayed on a heatmap and dendrogram.

\section{Counter Screen Assay}

Both the synaptogenesis assay and the Syngap1 expression assay rely on changes to the tdTomato signal. False positives may arise from the nonspecific effect of library compounds on the tdTomato signal; they may also have inherent fluorescence in the orange-red band used for signal detection in the primary assays. To detect these types of false positives, we developed an assay that quantifies nonspecific signals in the tdTomato emission spectrum. WT mouse primary neurons were cultured in 384-well assay plates using our standard HTS protocol and infected with an AAV vector (Penn vector \#AV-PV3365; $1 \times 10^{11}$ particles per plate) at DIV0 that drives tdTomato expression from the CAG promoter. Neurons were first imaged in the tdTomato channel at DIV12 followed by pinning of appropriate compounds or vehicle. All wells were imaged again at DIV14. At each time point, tdTomato-positive soma were segmented from acquired images and the average fluorescence intensity for all segmented soma in each well was calculated to derive a measure of tdTomato signal intensity. The assay endpoint was calculated by determining the ratio of DIV14/DIV12 tdTomato signal intensity for each well.

\section{Screening Details}

Library Details and Dosing Information. Pilot screens were carried out using the LOPAC1280 chemical library (Sigma, St. Louis, MO, USA) which is in DMSO. The LOPAC library was converted from a 96-well to a 384-well format, resulting in four plates with 320 compounds. We used the Biomek NX ${ }^{\mathrm{P}}$ pin tool with a 384 -well head to add library compounds immediately after imaging at DIV12. One hundred nanoliters of the 10-mM library compounds were pinned in each well. The final concentration of the compounds was $12.5 \mu \mathrm{M}$ in $80 \mu \mathrm{L}$ culture medium.

Plate Layout, Controls, and Biological Replicates. To aid in our understanding of reliability of the assay, the LOPAC library was screened in quadruplicate. The use of quadruplicate is both manageable and reduces potential errors. In addition, the minimal version of the assay discussed in the text also contributes to the reduction in variation by minimizing sample handling. Thus, this strategy required sixteen 384-well plates to screen the LOPAC library. For convenience, we split the screen into two batches (one litter = one batch) of 8 plates, and the batches were separated by 1 week. The plate layout was standard. Negative controls (0.125\% DMSO) were placed in columns $1,2,23$, and 24 in every screening plate in both screens, resulting in 64 negative control wells per plate and a total of 1,024 control wells per screen. We imaged Syn-TD punctate structures at DIV12 and DIV14. Compounds were added immediately after the DIV 12 read. The critical assay endpoint was the change in the number of detected synapses (i.e., the number of Syn-TD structures) per well between DIV12 and DIV14 (DIV14/ DIV12 on a per-well basis).

Improved Scalability of Neuron-Based Phenotypic Screening Assays
Image Analysis. For both screens, the dsRed/Cy3 channel images (Synaptophysin-tdTomato signals) were analyzed using the IN Cell 6000 software (GE Healthcare Life Sciences, Pittsburgh, PA, USA) using a custom puncta detection algorithm (available as an upload). The algorithm used "Object Segmentation" along with various classifiers (i.e., sensitivity, size/length, form factor, and threshold levels) to identify clearly isolated presynaptic structures.

Screening Data Normalization and Hit Threshold. Prior to data normalization, outliers in the DMSO control population that had a synaptic DIV14/DIV12 ratio $<1$ and fell outside of three stand deviations of the DMSO control mean were removed. Through extensive analysis, a ratio $<1$ indicated an issue with data collection (e.g., out-of-focus image), and these wells were therefore excluded. These corresponded to $14(1.4 \%)$ and 75 (7.3\%) out 1,024 DMSO control wells for screens s1 and s2, respectively. Despite the lack of evidence of plate variability in the assay (Fig. 1d), correction for plate-to-plate variability was done by normalizing the synaptic ratio DIV14/DIV12 of individual compounds to the mean of the synaptic ratio of DMSO controls within each plate. After data normalization, the mean of each four-replicate plate location was derived, and a hit threshold for compound above three standard deviations was applied. To further test for the possibility that edge effects impacted our primary hit identification, a $z$-score normalization was performed. The within-plate mean of synaptic ratio DIV14/DIV12 for all wells was subtracted from the individual compound ratio and divided by the standard deviation of all the wells within the plate. This resulted in similar preliminary hits within each screen when compared to DMSO control normalization.

\section{Results}

Neuron-based phenotypic assays are most often carried out in 96-well formats $[8,9]$. This low-density format severely limits assay scalability. Thus, it was unclear whether primary neuronal cultures were compatible with high-density assay plates used for large-scale screening projects. Therefore, we created a simplified culturing protocol which omitted all unnecessary steps, paired it with automated plating and maintenance workflows, and then measured both culture reliability and scalability. Cultures plated for HTS assays were viable and healthy, containing neurons with branched dendrites and numerous spines (Fig. 1a). We then cultured primary neurons from AI34D mice [10], which conditionally express the fusion protein Synaptophysin-tdTomato, a reliable marker of presynaptic terminals [11]. The Cre dependence of the reporter promotes assay flexibility by enabling the labeling of synapse subtypes (online suppl. Fig. 1a; for all online suppl. material, see www.karger.com/doi/10.1159/000481731) or by titrating the number of neurons that express the reporter (Fig. 1b). The latter, when combined with the appropriate viral titer of AAV-Cre at plating, greatly im- 
proves assay sensitivity by optimizing the density, and therefore detection, of isolated synaptic structures. As an objective measure of neuronal health and survival, we sampled both synapse and cellular density for every well by imaging Synaptophysin-tdTomato and NucBlue signals, respectively (online suppl. Fig. 1b). Cultured neurons performed equally well across different density assay plates (Fig. 1c; online suppl. Fig. 1c, d) and were scalable to high-density formats, including 1,536-well HTS-compatible plates, with little evidence of row or column effects in AI34D-based cultures (Fig. 1d). These data demonstrate that automated culturing of primary neurons is compatible with HTS-scale assay formats.

In general, HTS predominantly relies on the successful supply of mass quantities of robust, reproducible, and efficacious reagents and cell lines [12]. A potential limitation for the use of primary neurons for HTS-compatible assays is that the availability of biological material is limited by the frequency of litters. Even in best-case scenarios, primary neuron-based screening projects would require data collection across multiple culture "batches." Thus, a requirement for a primary neuron-based screening platform is that automated culturing procedures produce minimal batch-to-batch variance, with each batch providing a sufficient supply of primary neurons. To objectively measure batch variance, a "stress test" was carried out where we prepared AI34D primary neurons in sixty 384-well plates cultured as six distinct batches (1 litter $=1$ batch) prepared over the course of 3 weeks (online suppl. Fig. 2a). Synapse and cell density were sampled in every well. The completed experiment yielded $>23,000$ multiplexed data points, with the relationship between synapse density and cell density plotted for all wells (Fig. 1e). The relationship between the multiplexed measures was similar across the plates (Fig. 1e; online suppl.

Fig. 3. Development and validation of an HTS-compatible Syngap1 expression assay. a Schematic representation of the reporter insertion within the mouse Syngap1 locus and expected biology targeted by library molecules to change tdTomato/SynGAP expression levels. b Immunoblots of cortex homogenates from heterozygous Rum3 mice sacrificed at PND1 or PND21. tdTomato and SynGAP expression was normalized to a protein loading control to yield an RLU. The SynGAP groups had equal variance (Levene's test homogeneity of variance: $\mathrm{F}[1,6]=0.0487, p=0.832)$ and were normally distributed (Shapiro-Wilk test $\mathrm{P} 1 \mathrm{~W}=0.869, p=$ 0.296 , and $\mathrm{P} 21 \mathrm{~W}=0.831, p=0.170$ ); statistical analysis was done by unpaired two-sample $t$ test (two-tailed). P1 $(n=4)$ versus P21 $(n=4) t(6)=-29.614, p<0.001$. The tdTomato groups had equal variance equal (Levene's test homogeneity of variance: $F[1,6]=$
Fig. 2b), with a clear correlation between synapse and cellular densities. A cluster analysis of all sixty plates revealed eight major nodes, rather than the expected six if batches clustered among themselves, with most nodes containing plates derived from different batches (Fig. 1f). The median and distribution of the synapse/neuron ratio was similar among all plates (online suppl. Fig. 2c). Furthermore, the variance within each batch, as well as the variance among all batches, was $<20 \%$ (online suppl. Fig. 1d). Based on these data and former HTS experience [13], we concluded that automated procedures for producing primary neurons for screening purposes result in highly reproducible weekly culture conditions.

We next sought to create a disease-relevant phenotypic assay that highlights the strengths of a neuron-based screening platform. Loss of functional synapses is a hallmark of most brain disorders, such as neurodegeneration disorders and stroke [14], and contributes to impaired brain function during normal aging [15]. Chemical enhancement of neuronal communication by "boosting" functional synaptic connectivity is, therefore, a promising therapeutic strategy to generally improve brain function $[16,17]$. However, synapses are highly complex and unique to neurons. Thus, it is logical that the most effective screening assay would capture a disease-linked endpoint obtained from synaptically connected neurons. We sought to develop a phenotypic assay designed to broadly report an increase in synaptic connectivity in live primary neurons. Because of the ability to image synaptic structures in live primary neurons, our strategy was to perform an abbreviated kinetic assay where the total number of synaptic puncta were imaged in each well at multiple time points (Fig. 2a). This assay strategy had two significant advantages. First, by normalizing the second read to the first (i.e., DIV14/DIV12 ratio), the resulting ratio report-

$1.0325, p=0.3488$ ) and were normally distributed (Shapiro-Wilk test $\mathrm{P} 1 \mathrm{~W}=0.934, p=0.619$, and $\mathrm{P} 21 \mathrm{~W}=0.986, p=0.935$ ); statistical analysis was done by unpaired two-sample $t$ test (two-tailed) $\mathrm{P} 1(n=4)$ versus P21 $(n=4) t(6)=-7.78, p<0.001$. c Neurons from PND0 WT or heterozygous Rum 3 mice were cultured and imaged at DIV3. A simple intensity threshold was applied to illustrate the high contrast of signal from Rum-positive neurons. ii, integrated intensity of thresholded signal. d Plate-level metrics (soma intensity = black; coefficient of variation of soma intensity $=$ red) from negative control wells across the pilot screen. e Hit plots for individual negative control (left) or compound (right) well locations in the pilot screen; $n=4$ wells per data point. Black dots reflect the mean of the four replicates (red dots).

(For figure see next page.)
Spicer et al. 


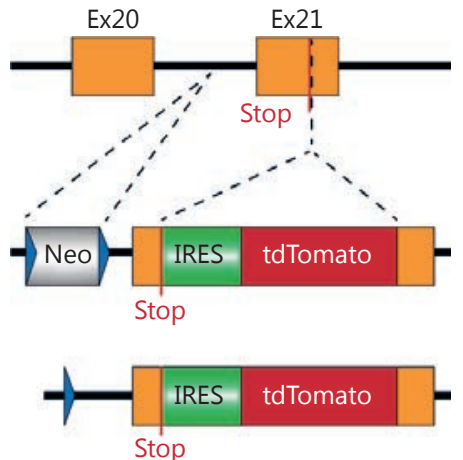

a Rum3-Syngap1 recombined locus
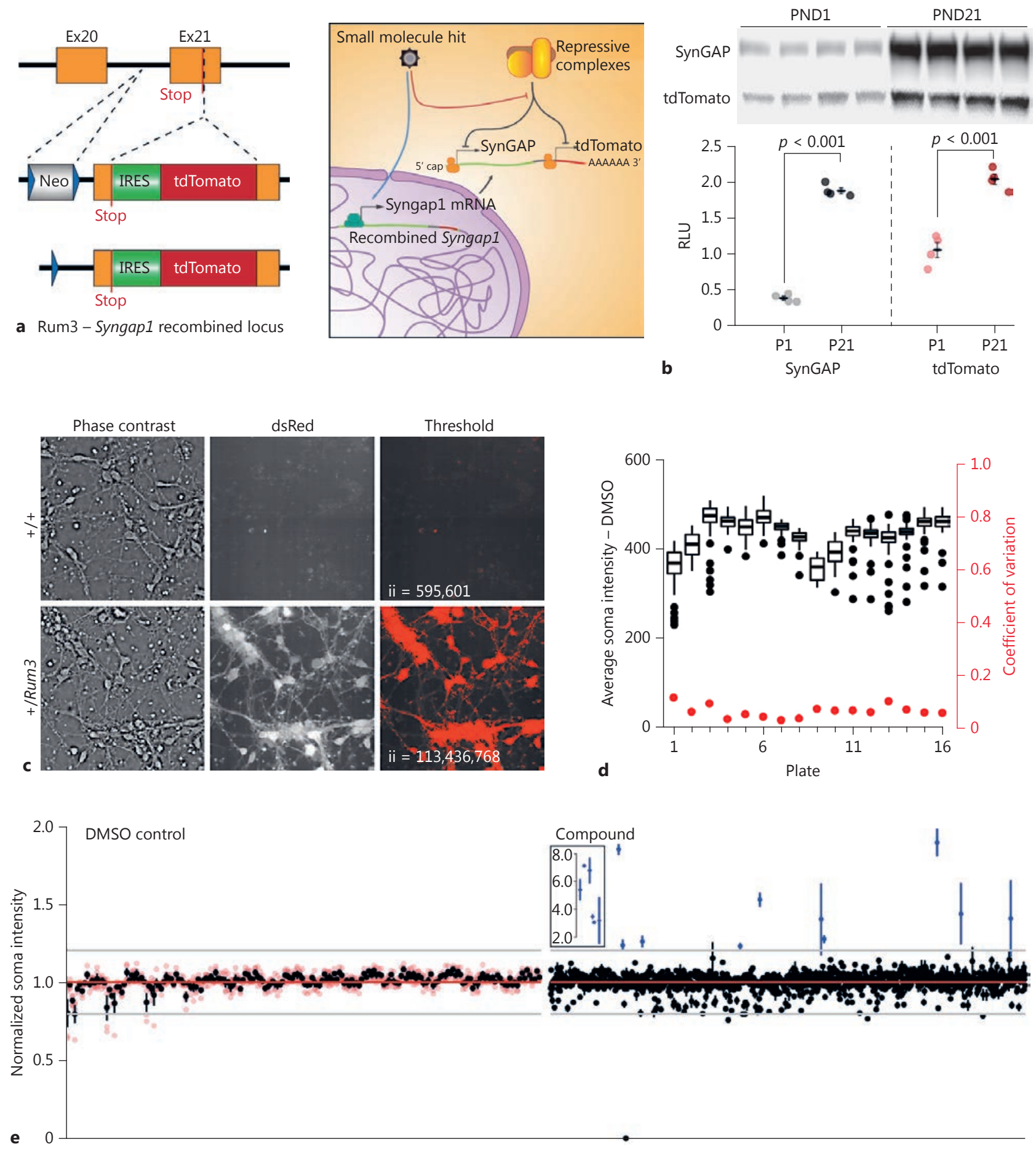
ed the rate of synaptogenesis during the assay period (Fig. 2b), a critical phenotype directly connected to neural circuit assembly. Second, the ratio was much less variable than puncta density (Fig. 2c), which is the assay endpoint typically used in synaptogenesis assays. Based on this finding, we designed a screening assay that utilized this abbreviated kinetic endpoint to discover compounds that enhance synaptogenesis rates in primary neurons (online suppl. Fig. 3a). We screened a library of 1,280 known pharmacologically active compounds (in quadruplicate) in a standard neurobiology laboratory environment, a strategy that required only two litters of AI34D mice. Analysis of DMSO controls (i.e., basal signal) indicated that the assay endpoint exhibited low variance within individual plates and repeatability among all sixteen plates used in the screen (online suppl. Fig. 3b, c). To identify preliminary hits, we performed two types of compound normalization methods, comparison of compound well measurements to 3 standard deviations of DMSO controls only [18], and $z$-score normalization to all wells in the plate on a plate-by-plate basis. These analyses yielded 9 and 10 candidates, respectively (online suppl. Table 1). The two lists were largely overlapping, which likely reflects the similarity of key assay parameters when applying each of these analyses.

We were interested in determining the reproducibility of preliminary hits identified in the assay. A nearly identical version of the synapse assay was implemented in a $\mu \mathrm{HTS}$ environment that utilizes industrial-scale robotics and an automated pipeline for data analysis and reporting. The only practical modification in the replicate screen was the change in environment (e.g., in the screening laboratory clean room), the type of robotic microscope (see Methods), and that the assay was fully automated (e.g., hands-free). The plate-level metrics of controls were similar to those in the initial laboratory-level screen (online suppl. Fig. 3d). Preliminary hits (Fig. 2e) were again identified using DMSO or whole-plate normalizations, with both methods yielding a largely overlapping list of candidates (online suppl. Table 2). We then compared preliminary hits from both screens. GYKI52466 was the only compound shared in each preliminary hit list from both screens and did not appear to cause cellular toxicity (online suppl. Fig. 3e). Interestingly, there were two other AMPAR antagonists present in the library, though these compounds failed to induce a change in the assay endpoint in both screening runs (Fig. 2f). We confirmed that GYKI had minimal activity in a counter screen that quantifies nonspecific changes in somatic tdTomato signal intensity (Fig. 2g). The identification of GYKI52466 in the replicate screen demonstrates that primary neuron-based assays can be implemented in an academic $\mu$ HTS environment, a capability that has enabled an ongoing, largescale screen for compounds that modify synaptic connectivity.

To demonstrate the flexibility of this platform, we designed another disease-relevant and HTS-scalable assay that would benefit from the use of primary neurons. Pathogenic Syngap1 variants cause a genetically defined form of intellectual disability [19] with epilepsy [20], termed MRD5, that accounts for up to $0.5-1 \%$ of severe childhood brain disorders [21, 22]. This disorder is caused by de novo SYNGAP1 mutations that induce genetic haploinsufficiency $[23,24]$. Probes that increase Syngap1 expression in neurons would target the root cause of MRD5. However, Syngap1 expression is heavily enriched in the brain and the protein is developmentally regulated [25], suggesting that there are neuron-specific regulatory mechanisms that control SynGAP expression. Thus, an assay for Syngap1 expression built from primary neurons would be expected to yield the most promising lead compounds. To create a Syngap1 expression assay, we engineered a mouse line that drives expression of tdTomato under the control of the endogenous Syngap1 gene (Fig. 3a), a design that is expected to report the level of actively translated Syngap1 mRNA. Mice that expressed the targeted insertion bred normally and we obtained viable heterozygous and homozygous offspring. Several lines of evidence indicated that tdTomato expression in neurons from this mouse line can report endogenous SynGAP levels. First, tdTomato fluorescence was observed in vivo, and the signal was proportional to the genotype (online suppl. Fig. 4a), demonstrating that the reporter itself expresses a dose-dependent linearity. Second, increased tdTomato expression during development paralleled that of endogenous SynGAP (Fig. 3b), and fluorescence within the dentate gyrus granule layer reflected the developmental age of neurons (online suppl. Fig. 4b). Third, primary neurons cultured from these mice expressed tdTomato with high contrast as early as postnatal day 3 (PND3) (Fig. 3c), and the critical assay endpoint (i.e., average fluorescent intensity of thresholded cell bodies; online suppl. Fig. 4c) was stable for the duration of the culture. Together, these data indicate that tracking of tdTomato signal dynamics in primary neurons from this reporter mouse would be an effective assay for identifying compounds that upregulate Syngap1 expression.

To test the performance of the SynGAP expression assay, we again screened the library of 1,280 known phar- 
macologically active compounds against the selected endpoint (i.e., average fluorescence intensity of tdTomatopositive soma; online suppl. Fig. 4c) using a design nearly identical to that of the synaptogenesis assay. The only notable exception was that the assay was carried out at DIV9 using a single read on a robotic confocal microscope. The average endpoint signal and the signal coefficient of variation for negative controls was consistent across plates used in the pilot screen (Fig. 3d), indicating that the assay endpoint exhibits low intrinsic noise. Consistent with this observation, we observed zero false positives in the negative control group (Fig. 3e). In contrast, we observed many preliminary hits from well locations representing library compounds (Fig. 3e; online suppl. Table 3). Given that we observed low intrinsic assay noise $(<10 \%$ coefficient of variation of controls) and several library compounds induced a robust change in tdTomato signal, the SynGAP expression assay appears to have a large dynamic range. Furthermore, since the assay features a simple, low-noise readout (i.e., tdTomato fluorescence in live neurons), we anticipate that it will scale well within a $\mu$ HTS environment.

\section{Discussion}

The ability to successfully implement multiple primary neuron-based assays in both low- and high-throughput screening environments is evidence of this platform's flexibility and scalability. Perhaps most importantly, we found that assays created using this platform are economical. Our studies demonstrate that there is enough material in a single litter of mice to screen 1,000 or more compounds. Thus, once an assay is validated, it is possible to screen thousands of compounds in primary neurons as a stand-alone project in a laboratory environment. Indeed, the essential elements of our screening runs were carried out on equipment that already exists in many academic laboratories or imaging cores. As a result, both assay development and screening steps can be carried out by individual investigators in their own laboratory, while the most promising assays can be implemented in a true HTS environment for much larger-scale screens. A significant advantage of this assay platform, which is based on knockin mice, is that in vivo biological validation of preliminary hits can be carried out in the same animals that supply material for in vitro assays. Taken together, the combined features of this platform improve the accessibility of drug discovery within the neuroscience community, which could stimulate drug discovery in CNS disorders. Future

Improved Scalability of Neuron-Based Phenotypic Screening Assays improvements in this platform will include the incorporation of iPSC-derived human neurons, which will complement assays performed in nonhuman primary cells, with respect to secondary assays used to biologically validate lead compounds.

For successful long-term screening projects that utilize primary cells in an automated environment, it is essential to obtain simple Go/NoGo measures in order to maintain quality during the entire project period. Unlike established cell lines, primary cells obtained for screening purposes are derived from animal models. Thus, a protracted screening project will utilize cells derived from different animals over time, which could influence the biology of the cells, potentially degrading assay performance. In one such case, we recently performed a 36week automated synaptogenesis screen that assayed $\sim 80,000$ neuronal culture wells. To maintain assay performance across the entire project, we implemented strict quality control metrics that served as a Go/NoGo criterion for each weekly batch of primary neurons. During assay validation steps, we identified several simple plate-level measures that predicted performance of the desired assay endpoint. In the synaptogenesis assay, we found that the average number of detected synapses per well, and the variance of synapses across all wells of a plate at DIV8, correlated well with the overall synaptogenesis rate and synaptogenesis variance of the same plate from DIV12-DIV14 (our desired endpoint). Analyzing dozens of batches allowed us to determine a range of acceptable values at DIV8, which then served as Go/ NoGo criteria for collecting screening data with any particular batch. To implement this strategy into the ongoing screening project, we created one additional plate per batch that served as a "sentinel," which was rapidly imaged on a laboratory-level instrument at DIV8 in order to obtain the Go/NoGo metric. This strategy was possible because our analysis during assay validation demonstrated that plates within batches performed similarly (online suppl. Fig. 2). If the sentinel passed this test, the batch was delivered to the screening facility. An added benefit of this laboratory-based sentinel strategy was that it accelerated robotic implementation. The sentinel plate was always saved until the batch run was finished. If substandard data were obtained in the automated facility, the sentinel plate was checked for data integrity in the laboratory, which helped identify the cause of substandard data collection. This quality control protocol helped identify a step in the automated procedure that increased the risk for culture contamination in addition to assuring weekly assay performance.

Mol Neuropsychiatry 2017;3:141-150 149 


\section{Acknowledgment}

This work was supported by NINDS grant R21/33 NS082640.

\section{Statement of Ethics}

Housing, animal care, and experimental procedures were consistent with the Guide for the Care and Use of Laboratory Animals and approved by the Institutional Animal Care and Use Committee.

\section{Disclosure Statement}

The authors have no competing financial interests to declare.

\section{Author Contributions}

C.H., T.V., D.C., C.R., M.K., K.V., F.M., P.B., and J.S. performed the research; T.P.S., K.A.M., D.T.P., S.P., P.H., R.D., C.A.M., L.S., and G.R. designed the research; T.P.S., C.H., T.V., D.C., L.S., and G.R. analyzed the data; T.P.S., T.V., C.A.M., and L.S. edited the manuscript; G.R. wrote the manuscript.

\section{References}

1 Dean B, Moller HJ, Svensson TH, Geyer MA, Rujescu D, Scarr E, Millan MJ: Problems and solutions to filling the drying drug pipeline for psychiatric disorders: a report from the inaugural 2012 CINP Think Tank. Int J Neuropsychopharmacol 2014;17:137-148.

2 Brady LS, Insel TR: Translating discoveries into medicine: psychiatric drug development in 2011. Neuropsychopharmacology 2012;37: 281-283.

3 Eder J, Sedrani R, Wiesmann C: The discovery of first-in-class drugs: origins and evolution. Nat Rev Drug Discov 2014;13:577-587.

4 Mullard A: The phenotypic screening pendulum swings. Nat Rev Drug Discov 2015;14: 807-809.

5 Prior M, Chiruta C, Currais A, Goldberg J, Ramsey J, Dargusch R, Maher PA, Schubert $\mathrm{D}$ : Back to the future with phenotypic screening. ACS Chem Neurosci 2014;5:503-513.

6 Vincent F, Loria P, Pregel M, Stanton R, Kitching L, Nocka K, Doyonnas R, Steppan C, Gilbert A, Schroeter T, Peakman MC: Developing predictive assays: the phenotypic screening "rule of 3." Sci Transl Med 2015; 293 ps15.

7 Zheng W, Thorne N, McKew JC: Phenotypic screens as a renewed approach for drug discovery. Drug Discov Today 2013;18:10671073.

8 Sharma K, Choi SY, Zhang Y, Nieland TJ, Long S, Li M, Huganir RL: High-throughput genetic screen for synaptogenic factors: identification of LRP6 as critical for excitatory synapse development. Cell Rep 2013;5:13301341.

9 Nieland TJ, Logan DJ, Saulnier J, Lam D, Johnson C, Root DE, Carpenter AE, Sabatini BL: High content image analysis identifies novel regulators of synaptogenesis in a highthroughput RNAi screen of primary neurons. PLoS One 2014;9:e91744.

10 Madisen L, Zwingman TA, Sunkin SM, Oh SW, Zariwala HA, Gu H, Ng LL, Palmiter RD, Hawrylycz MJ, Jones AR, Lein ES, Zeng H: A robust and high-throughput Cre reporting and characterization system for the whole mouse brain. Nat Neurosci 2010;13:133-140.
11 Harms KJ, Tovar KR, Craig AM: Synapsespecific regulation of AMPA receptor subunit composition by activity. J Neurosci 2005;25: 6379-6388.

12 Dunne A, Jowett M, Rees S: Use of primary human cells in high-throughput screens. Methods Mol Biol 2009;565:239-257.

13 Smith E, Janovick JA, Bannister TD, Shumate J, Scampavia L, Conn PM, Spicer TP: Identification of potential pharmacoperones capable of rescuing the functionality of misfolded vasopressin 2 receptor involved in nephrogenic diabetes insipidus. J Biomol Screen 2016;21:824-831.

14 Canter RG, Penney J, Tsai LH: The road to restoring neural circuits for the treatment of Alzheimer's disease. Nature 2016;539:187196.

15 Morrison JH, Baxter MG: The ageing cortical synapse: hallmarks and implications for $\operatorname{cog}$ nitive decline. Nat Rev Neurosci 2012;13: 240-250.

16 Rumbaugh G, Sillivan SE, Ozkan ED, Rojas CS, Hubbs CR, Aceti M, Kilgore M, Kudugunti S, Puthanveettil SV, Sweatt JD, Rusche J, Miller CA: Pharmacological selectivity within class I histone deacetylases predicts effects on synaptic function and memory rescue. Neuropsychopharmacology 2015;40: 2307-2316.

17 Lu B, Nagappan G, Guan X, Nathan PJ, Wren $\mathrm{P}$ : BDNF-based synaptic repair as a diseasemodifying strategy for neurodegenerative diseases. Nat Rev Neurosci 2013;14:401-416.

18 Zhang JH, Chung TD, Oldenburg KR: A simple statistical parameter for use in evaluation and validation of high throughput screening assays. J Biomol Screen 1999;4:67-73.

19 Hamdan FF, Gauthier J, Spiegelman D, Noreau A, Yang Y, Pellerin S, Dobrzeniecka S, Cote M, Perreau-Linck E, Carmant L, D'Anjou G, Fombonne E, Addington AM, Rapoport JL, Delisi LE, Krebs MO, Mouaffak F, Joober R, Mottron L, Drapeau P, Marineau C, Lafreniere RG, Lacaille JC, Rouleau GA, Michaud JL; Synapse to Disease Group: $\mathrm{Mu}$ tations in SYNGAP1 in autosomal nonsyndromic mental retardation. N Engl J Med 2009;360:599-605.
20 Carvill GL, Heavin SB, Yendle SC, McMahon JM, O'Roak BJ, Cook J, Khan A, Dorschner MO, Weaver M, Calvert S, Malone S, Wallace G, Stanley T, Bye AM, Bleasel A, Howell KB, Kivity S, Mackay MT, Rodriguez-Casero V, Webster R, Korczyn A, Afawi Z, Zelnick N, Lerman-Sagie T, Lev D, Moller RS, Gill D, Andrade DM, Freeman JL, Sadleir LG, Shendure J, Berkovic SF, Scheffer IE, Mefford HC: Targeted resequencing in epileptic encephalopathies identifies de novo mutations in CHD2 and SYNGAP1. Nat Genet 2013;45: 825-830.

21 Deciphering Developmental Disorders Study: Large-scale discovery of novel genetic causes of developmental disorders. Nature 2015;519: 223-228.

22 Deciphering Developmental Disorders Study: Prevalence and architecture of de novo mutations in developmental disorders. Nature 2017;542:433-438.

23 Mignot C, von Stülpnagel C, Nava C, Ville D, Sanlaville D, Lesca G, Rastetter A, Gachet B, Marie Y, Korenke GC, Borggraefe I, Hoffmann-Zacharska D, Szczepanik E, RudzkaDybała M, Yiş U, Çağlayan H, Isapof A, Marey I, Panagiotakaki E, Korff C, Rossier E, Riess A, Beck-Woedl S, Rauch A, Zweier C, Hoyer J, Reis A, Mironov M, Bobylova M, Mukhin K, Hernandez-Hernandez L, Maher B, Sisodiya S, Kuhn M, Glaeser D, Weckhuysen S, Myers CT, Mefford HC, Hörtnagel K, Biskup S; EuroEPINOMICS-RES MAE working group, Lemke JR, Héron D, Kluger G, Depienne C: Genetic and neurodevelopmental spectrum of SYNGAP1-associated intellectual disability and epilepsy. J Med Genet 2016;53:511-522.

24 Parker MJ, Fryer AE, Shears DJ, Lachlan KL, McKee SA, Magee AC, Mohammed S, Vasudevan PC, Park SM, Benoit V, Lederer D, Maystadt I, Study D, FitzPatrick DR: De novo, heterozygous, loss-of-function mutations in SYNGAP1 cause a syndromic form of intellectual disability. Am J Med Genet A 2015; 167A:2231-2237.

25 Kim JH, Liao D, Lau LF, Huganir RL: SynGAP: a synaptic RasGAP that associates with the PSD-95/SAP90 protein family. Neuron 1998;20:683-691. 